\title{
Terahertz master-oscillator power-amplifier quantum cascade lasers
}

\author{
Huan Zhu, ${ }^{1,2}$ Fangfang Wang, ${ }^{1}$ Quan Yan, ${ }^{1}$ Chenren Yu, ${ }^{1,2}$ Jianxin Chen, ${ }^{1}$ Gangyi Xu, ${ }^{1, a)}$ \\ $\mathrm{Li} \mathrm{He},{ }^{1}$ Lianhe Li, ${ }^{3}$ Li Chen, ${ }^{3}$ A. Giles Davies, ${ }^{3}$ Edmund H. Linfield, ${ }^{3}$ Jiaming Hao, ${ }^{4}$ \\ Pierre-Baptiste Vigneron, ${ }^{5}$ and Raffaele Colombelli ${ }^{5}$ \\ ${ }^{1}$ Key Laboratory of Infrared Imaging Materials and Detectors, Shanghai Institute of Technical Physics, \\ Chinese Academy of Sciences, Shanghai 200083, China \\ ${ }^{2}$ University of Chinese Academy of Sciences, Beijing 100049, China \\ ${ }^{3}$ School of Electronic and Electrical Engineering, University of Leeds, Leeds LS2 9JT, United Kingdom \\ ${ }^{4}$ National Laboratory of Infrared Physics, Shanghai Institute of Technical Physics, Chinese Academy \\ of Sciences, Shanghai 200083, China \\ ${ }^{5}$ Centre for Nanoscience and Nanotechnology (C2N Orsay), CNRS UMR9001, Univ. Paris Sud, Univ. Paris \\ Saclay, 91405 Orsay, France
}

(Received 24 August 2016; accepted 15 November 2016; published online 7 December 2016)

\begin{abstract}
We report on the realization of a monolithically integrated master-oscillator power-amplifier architecture in a terahertz quantum cascade laser (THz-QCL) with a metal-metal waveguide. The master-oscillator section is a first-order distributed feedback (DFB) laser. Instead of using a thick anti-reflection coating, we exploit a diffraction grating together with an absorbing boundary in the power-amplifier section to efficiently extract the laser radiation and suppress the self-lasing in it. The devices demonstrate a stable generation and power amplification of single-mode emission. The amplification factor is about 5 , and the output power is approximately twice that of the standard second-order DFB lasers fabricated from the same material. Emission beam pattern with a divergence angle of $\sim 18 \times 40^{\circ}$ is achieved. Our work provides an avenue for the realization of singlemode THz-QCLs with high output power and good beam quality. Published by AIP Publishing.

[http://dx.doi.org/10.1063/1.4969067]
\end{abstract}

Terahertz quantum cascade lasers (THz-QCLs) with single-mode emission are highly desired for applications such as spectroscopy, trace gas detection, and free space communication. $^{1-3}$ Intensive studies have been devoted to develop unique photonic coupling structures in order to approach a high output power and a directional beam pattern for singlemode THz-QCLs. ${ }^{4-17}$ Related results include third-order distributed feedback (DFB) lasers, ${ }^{5,6}$ second-order DFB THzQCLs with dual slits, ${ }^{7}$ THz-QCLs with a graded photonic heterostructure resonator, ${ }^{9-11}$ phase-locked arrays of secondorder DFB lasers, ${ }^{12-14}$ etc. However, to ensure that lasing occurs on transverse and longitudinal single-mode, the dimensions of the photonic coupling structure must be limited, thus restricting the available output power and increasing the divergence of the emitted beam.

Monolithically integrated master-oscillator power-amplifier (MOPA) is a promising architecture to realize single-mode emission with a high output power and a near diffractionlimited beam quality. It has been implemented in near-infrared diode lasers and also in mid-infrared quantum cascade lasers. ${ }^{18-22}$ In order to suppress the self-lasing in the poweramplifier (PA) section and to fully exploit the material gain, the front facet of the PA section needs to be coated by antireflection (AR) layers. However, extending the MOPA architecture to the $\mathrm{THz}$ frequency range is a challenge because of the lack of suitable AR coatings.

Only a few researches related to MOPA devices based on THz quantum cascade structures have been reported. ${ }^{23,24}$ In these devices-which are based on a single plasmon waveguide - the master oscillator (MO) section is a Fabry-

${ }^{a)}$ Corresponding author. E-mail: gangyi.xu@mail.sitp.ac.cn
Pérot laser and is separated from the PA section by a deep air gap. The PA section is another Fabry-Pérot cavity with one facet angled or coated by a thick parylene layer. The Fabry-Pérot MO section intrinsically results in multimode emission, while the deep air gap degrades the coupling efficiency of the $\mathrm{THz}$ radiation from the MO to the PA section. Also, for high temperature operation, a metal-metal waveguide is more desired but it, in turn, results in high facet reflection and thus facilitates self-lasing in the PA section. Because of these issues, the functionalities of the MOPA structure have not been fully exploited in THz-QCLs.

In this letter, we report monolithically integrated $\mathrm{THz}$ master-oscillator power-amplifier quantum cascade lasers (THz-MOPA-QCLs) that exhibit stable generation and the power amplification of single mode emission. Fig. 1(a) shows the scheme of a THz-MOPA-QCL. The device is based on a metal-metal waveguide, where both the $\mathrm{MO}$ and the PA sections share the same active region and are monolithically integrated on the same chip. The MO section is a first-order DFB laser, in which the metallic grating is formed on the top of the active region. The PA section consists of three parts: a power preamplifier, a light extraction part, as well as an absorbing boundary. The preamplifier linked to the MO is essentially a metal-metal waveguide where the active region is sandwiched between the two metallic layers. A diffraction grating is formed in the top metallization of the light extraction part where the $\mathrm{THz}$ wave is amplified and simultaneously extracted out of the device in an oblique direction. The emission direction is determined by the periodicity of the diffraction grating. The judicious design of the diffraction grating is implemented to minimize the reflection of the $\mathrm{THz}$ wave and to maximize the efficiency of the light 
(a)

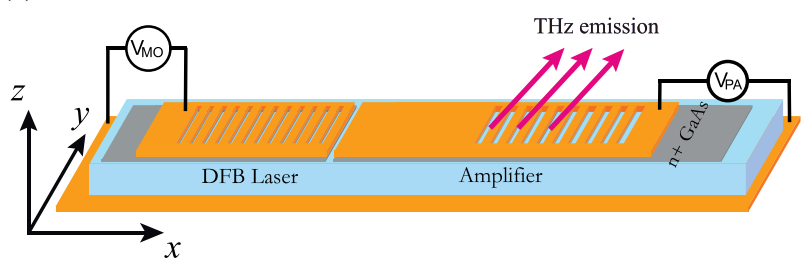

(b)

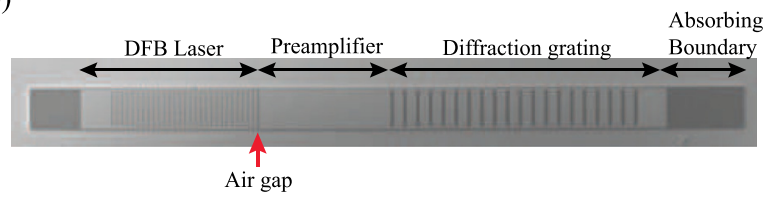

(c)

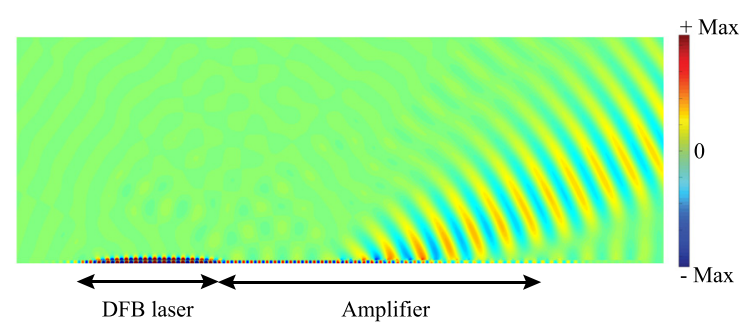

FIG. 1. (a) The schematic illustration of a THz-MOPA-QCL based on a metal-metal waveguide. The MO section is a first-order DFB laser. The PA section contains a preamplifier, a diffraction grating to extract the THz radiation, and an absorbing boundary. (b) The SEM image of a THz-MOPAQCL. The periodicity and the air slit width of the DFB grating are $20.6 \mu \mathrm{m}$ and $5 \mu \mathrm{m}$, respectively. The length of preamplifier is $500 \mu \mathrm{m}$, and the periodicity and the air slit width of the diffraction grating is $50 \mu \mathrm{m}$ and $15 \mu \mathrm{m}$, respectively. The DFB grating and the diffraction grating contain, respectively, 30 and 20 periods. The ridge width is $150 \mu \mathrm{m}$. The absorbing boundary is $400 \mu \mathrm{m}$ in length. (c) Mode distribution $\left(E_{z}\right)$ along the cross-section ( $x z$ plane) of the device.

extraction. The absorbing boundary features an uncovered $\mathrm{n}^{+}$GaAs contact layer which efficiently absorbs the $\mathrm{THz}$ wave transmitted through the diffraction grating. A diffraction grating with low reflectivity and a boundary with high absorption efficiency are crucial to suppress the self-lasing in the PA section. A narrow gap is formed in the top metallization between the MO and the PA sections which allows them to be separately pumped.

The QCL structure employed in this work is based on a bound-to-continuum active region. ${ }^{25}$ The $\mathrm{Al}_{0.15} \mathrm{Ga}_{0.85} \mathrm{As} /$ GaAs layer sequence in each stage of the active region is 3.8/14.0/0.6/9.0/0.6/15.8/1.5/12.8/1.8/12.2/2.0/12.0/2.0/11.4/ 2.7/11.3/3.5/11.6 (nm). AlGaAs layers are shown in bold, and the underlined GaAs layers are $n$ doped at a level of $1.6 \times 10^{16} \mathrm{~cm}^{-3}$. The laser core consists of 90 stages with a total thickness of $\approx 12 \mu \mathrm{m}$, and a measured central emission frequency of $\approx 2.5 \mathrm{THz}$. The fabrication of THz-MOPAQCLs is similar to that of second-order THz DFB lasers with a metal-metal waveguide. ${ }^{9}$ The top metallization, together with the metallic gratings and the $8-\mu \mathrm{m}$-wide air gap, was defined on the top of the active core by contact optical lithography followed by metal deposition and lift-off. The $\mathrm{n}^{+}$GaAs contact layer under the air gap and air slits was removed by inductively coupled plasma etching. The position of the air gap is indicated by an arrow in Fig. 1(b). The center-to-center distance between the air gap and the neighbor air slit equals to the DFB grating periodicity, so the air gap will not disturb the mode oscillation in the MO section or affect the wave injection from the MO to the PA section. The DFB grating and the diffraction grating contain, respectively, 30 and 20 periods, and the preamplifier part is $500 \mu \mathrm{m}$ in length. A series of THz-MOPA-QCLs with different $\Lambda_{D F B}$ (the periodicity of DFB grating) were fabricated. More device parameters are given in the caption of Figure 1. Fig. 1(b) shows a SEM image of a THz-MOPA-QCL.

We first theoretically investigate the MOPA concept in our structure. Fig. 1(c) plots the mode distribution in the device calculated by the $2 \mathrm{D}$ finite-difference time-domain (FDTD) method.

By carefully designing the periodicity and the duty cycle of the DFB grating, we make only the DFB band edge mode above the photonic band gap located in the frequency range of the material gain. Fig. 1(c) shows the mode oscillation in the MO section, the injection and the propagation of the $\mathrm{THz}$ wave in the PA section, as well as the wave extraction. To avoid the field divergence, the material gain is not considered in the calculation, so the power amplification is not presented in Fig. 1(c). Full-wave finite element method (FEM) simulations are then carried out to investigate the power amplification and the suppression of the self-lasing in the PA section. We have calculated the reflection, diffraction, and transmission of the $\mathrm{THz}$ wave with a different frequency when it is injected into the PA section. For clarity, the measured emission spectrum of a THz-MOPA-QCL is shown in Fig. 2(a), illustrating the frequency of the DFB mode. The calculated results are given in Figs. 2(b) and 2(c), where the net material gain is set as 0 and $20 \mathrm{~cm}^{-1}$, respectively. Fig. 2(b) shows the case of a transparent waveguide, in which, the two reflectivity plateaus correspond to the two photonic band gaps of the diffraction grating. The frequency range of the laser emission, marked by the green shadowed region in Fig. 2(b), locates in-between the band gaps and the related reflectivity is negligible. The diffraction efficiency is more than $90 \%$ in the frequency range from 2.4 to $2.6 \mathrm{THz}$, which indicates that most of the $\mathrm{THz}$ wave emitted from the MO section can be collected. When the net material gain is $20 \mathrm{~cm}^{-1}$, as shown in Fig. 2(c), the calculated reflectivity is still lower than $3 \times 10^{-2}$ in the frequency range of the material gain. The power of the diffracted radiation is about 5 times than that injected into the PA section, indicating an amplification efficiency of $\sim 5$. Due to the material gain, the power of the $\mathrm{THz}$ wave transmitted through the diffraction grating is comparable to that injected into the PA section. For this reason, a 400- $\mu$ m-long $\mathrm{n}^{+}$GaAs absorbing boundary is exploited to absorb the transmitted $\mathrm{THz}$ wave. After all, the low calculated reflectivity indicates that the self-lasing in the PA section can be suppressed, and the perturbation of the reflected $\mathrm{THz}$ wave on the field oscillation in the MA section is also negligible.

The fabricated THz-MOPA-QCLs were measured at $20 \mathrm{~K}$ in the pulsed mode with a repeated frequency of $25 \mathrm{kHz}$ and a pulse width of $1 \mu \mathrm{s}$. Fig. 3(a) presents the emission spectra of 3 THz-MOPA-QCLs in which $\Lambda_{D F B}$ varies from $20.4 \mu \mathrm{m}$ to $20.8 \mu \mathrm{m}$. Single-mode emission is achieved, and the wavelength scales linearly with the grating periodicity. The devices exhibit a stable single-mode emission in the whole dynamic range of the MO and the PA sections. Fig. 
(a)

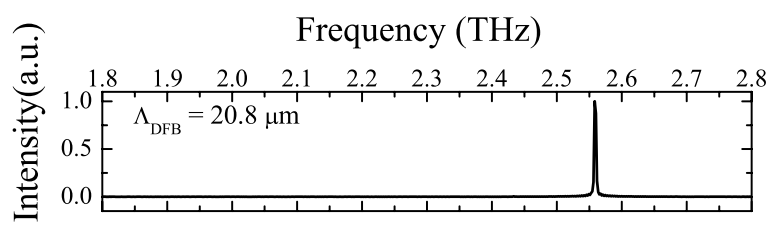

(b)

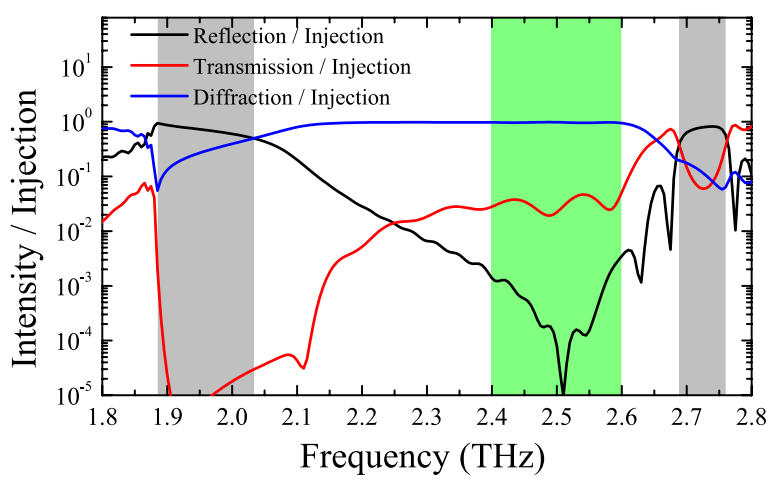

(c)

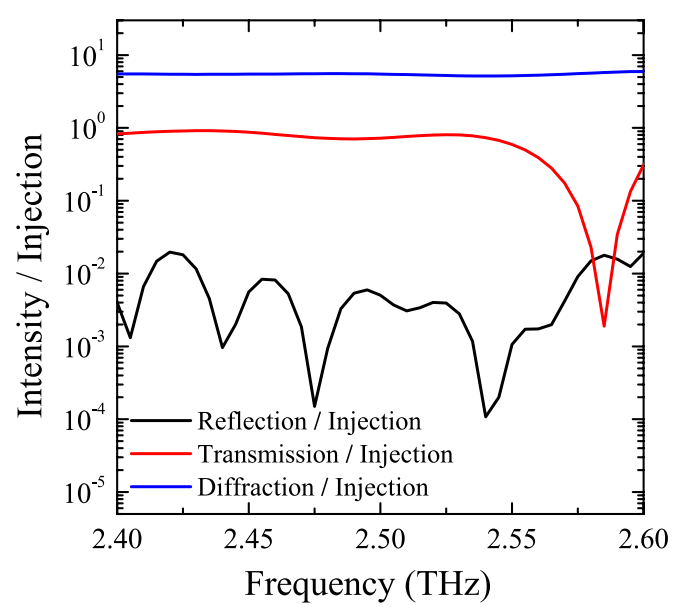

FIG. 2. (a) Emission spectrum of a THz-MOPA-QCL. (b) and (c) show the calculated intensity of the reflected, transmitted, and diffracted waves caused by the diffraction grating, divided by the intensity of the $\mathrm{THz}$ wave injected into the PA section. In panel (b), the structure is set as transparent. The 2 gray shadowed regions correspond to the photonic band gaps of the diffraction grating. The green region shows the frequency range of the laser emission. In panel (c), the material gain is set as $20 \mathrm{~cm}^{-1}$.

3(a) also shows that no self-lasing was observed when only the PA section was biased above the threshold. The effective refractive index of the operation mode is 2.83 , deduced from the grating periodicity and the emission wavelength. Comparing with the FDTD calculations, we find the refractive index of the active region is about 3.49 , very close to the value (3.50) calculated from the low temperature refractive indexes of the related materials and the Drude model. ${ }^{26}$ The low effective index of the operation mode is caused by the penetration of the electromagnetic field through the air slits. These results demonstrate that the device operates on the DFB mode, and the $\mathrm{THz}$ emission is stimulated in the MO section. Fig. 3(b) shows the emission spectrum of a typical THz-MOPA-QCL when the MO and the PA sections are equally biased and the output power reaches its maximum, demonstrating that the side mode suppression ratio is about $30 \mathrm{~dB}$.
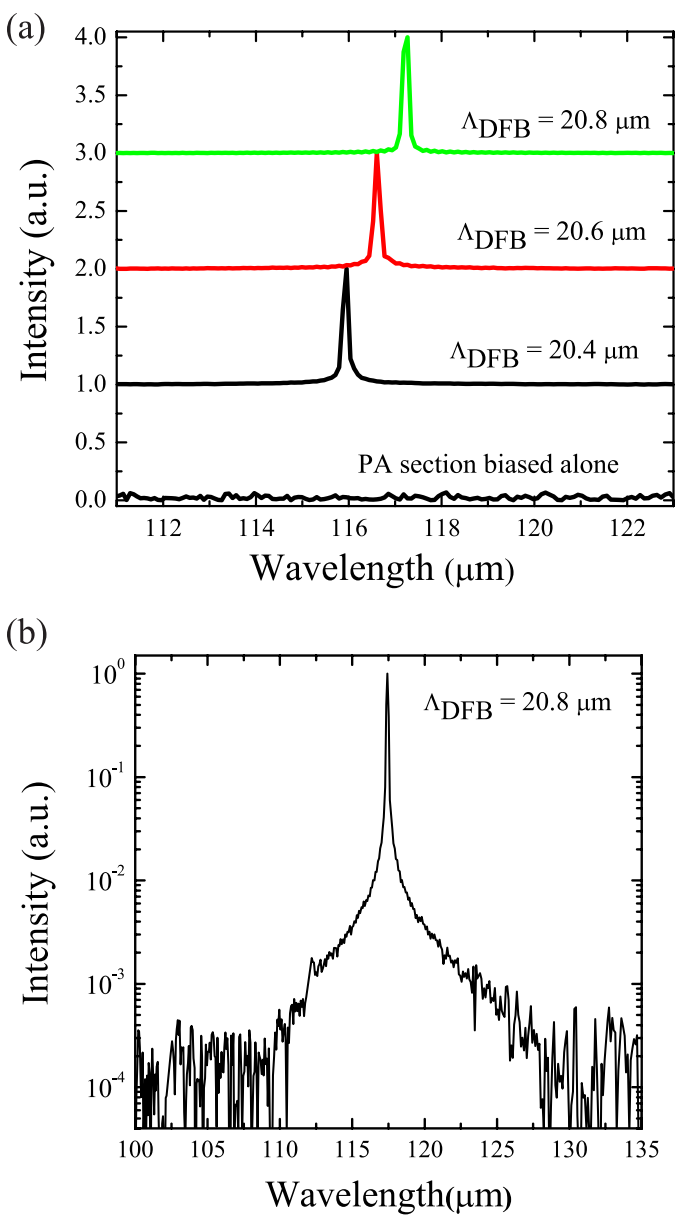

FIG. 3. (a) The emission spectra of 3 THz-MOPA-QCLs measured at $20 \mathrm{~K}$ in pulsed mode, where $\Lambda_{\mathrm{DFB}}$ varies from $20.4 \mu \mathrm{m}$ to $20.8 \mu \mathrm{m}$. Spectral result where only the PA section is biased above the threshold is also shown, and no self-lasing is observed. (b) Emission spectrum of a typical THz-MOPAQCL when the MO and PA sections are equally biased at the level of the maximum output power.

The far-field emission patterns of the lasers were measured at $20 \mathrm{~K}$ with a Golay cell detector, which was scanned on a 15$\mathrm{cm}$-radius sphere centered on the device surface. Fig. 4(a) shows schematically the measurement geometry. Fig. 4(b) shows the measured far-field emission pattern of a typical device, where both the MO and the PA sections are equally biased at the level of maximum output power. The emission direction of the highest brightness is $25^{\circ}$ deviated from the plane of the device surface $\left(\theta_{x}=25^{\circ}\right)$, and the full width at half maximum (FWHM) of the beam pattern is $\sim 18^{\circ} \times 40^{\circ}$. Fig. 4(c) shows the calculated emission pattern by FDTD simulations, in a good agreement with the measured results. The simulation indicates the lateral ends of the air slits in the diffraction grating induced light scattering, which results in the two emission maximums at $\theta_{y}$ $\approx \pm 10^{\circ}$. Recently, emission beam with the very low divergence angle $\left(<10 \times 10^{\circ}\right)$ has been demonstrated in a phased array of THz-QCLs and a THz-QCL vertical-external-cavity surfaceemitting-laser. ${ }^{13,16}$ In the THz-MOPA-QCL, the beam divergence is determined by the dimensions of the emission aperture. Therefore, a tapered PA section with a widened diffraction grating will efficiently decrease the divergence angle.

Fig. 5(a) shows the light-current-voltage $(L-I-V)$ characteristics of a typical THz-MOPA-QCL measured at $20 \mathrm{~K}$, where the output power was measured as a function of the 
(a)

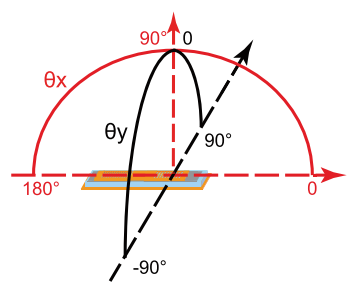

(b)

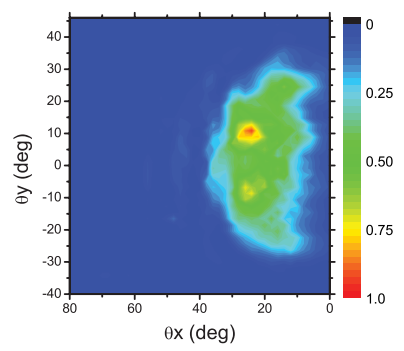

(c)

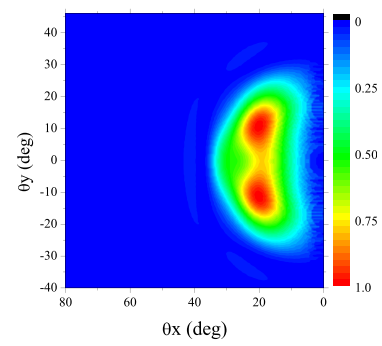

FIG. 4. (a) Schematic diagram defining the scanning angles used in beamprofiling measurements. The angle $\theta \mathrm{x}=\theta \mathrm{y}=0$ corresponds to the direction along the laser ridge. (b) and (c) show the measured and calculated farfield patterns of a THz-MOPA-QCL, respectively.
MO current at various PA bias $\left(\mathrm{V}_{\mathrm{PA}}\right)$. When the PA section is biased at the laser threshold $\left(\mathrm{V}_{\mathrm{PA}}=3.41 \mathrm{~V}\right)$, where it can be approximately regarded as a transparent media, the device peak output power is $0.26 \mathrm{~mW}$. When the PA bias is $4.30 \mathrm{~V}$, corresponding to the peak gain available, the peak output power reaches $1.32 \mathrm{~mW}$. As a simple estimation, the amplification factor is about 5. According to the calculated results presented in Fig. 2(b), the maximum net material gain in our metal-metal waveguide is about $20 \mathrm{~cm}^{-1}$.

For performance comparison, we fabricated the MO section alone as a first-order DFB laser, but the measurements reveal that it operates in multi-mode and the output power is much less than that of the MOPA device. The multi-mode emission may be caused by the high facet reflectivity, and
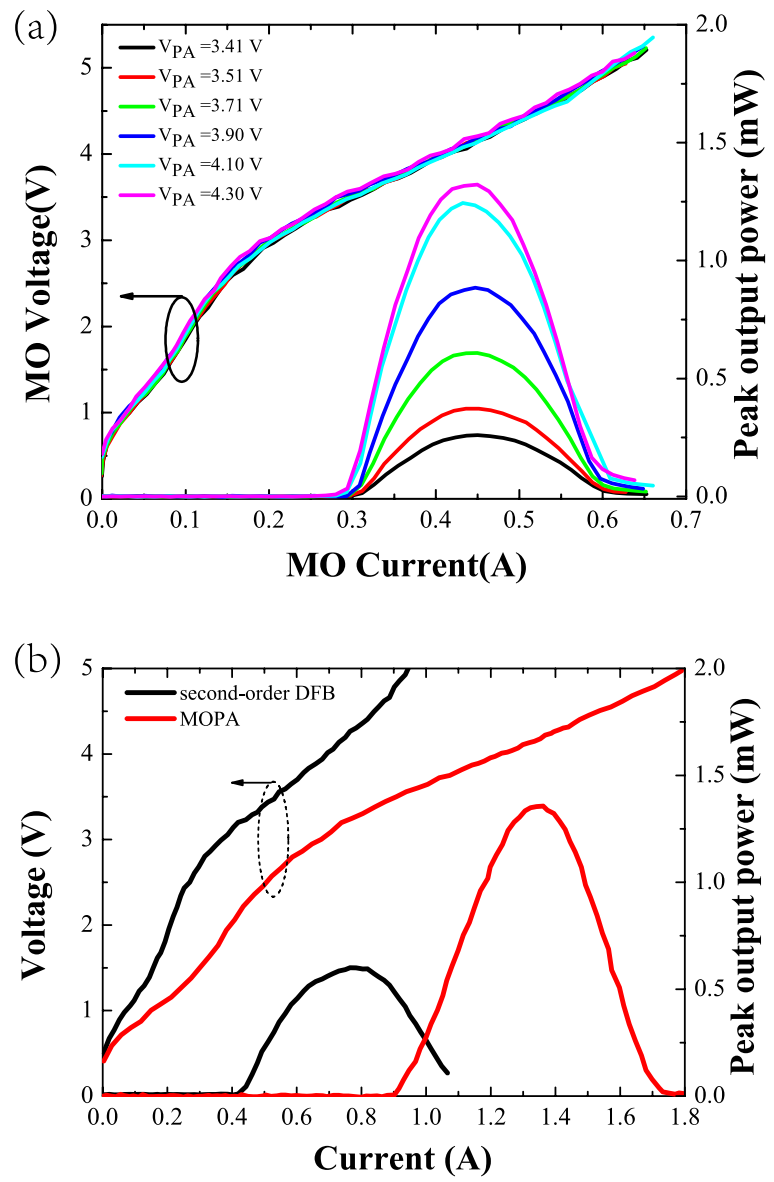

FIG. 5. (a) Light-current-voltage ( $L-I-V)$ curves of a typical THz-MOPAQCL measured at $20 \mathrm{~K}$ in the pulsed mode. The output power is measured as a function of the MO current when the PA section is biased at a different level $\left(\mathrm{V}_{\mathrm{PA}}\right)$. (b) $L-I-V$ curves of a second-order THz DFB laser and a THzMOPA-QCL in which the MO and the PA sections are equally biased. Both are measured at $20 \mathrm{~K}$ in the pulsed mode. the low output power is mainly related to the highly divergent beam pattern and the short cavity length $(\sim 0.7 \mathrm{~mm})$.

We therefore compare the THz-MOPA-QCLs with second-order DFB lasers fabricated from the same material, since the latter have been intensively studied and have very similar fabrication processes. The periodicity, the number of periods, and the duty cycle of the second-order DFB grating are, respectively, $33.4 \mu \mathrm{m}, 40$, and $85 \%$. The ridge width and the cavity length of the second-order DFB laser are $150 \mu \mathrm{m}$ and $1.4 \mathrm{~mm}$, respectively. The DFB grating parameters are optimized according to the previous research in order to maximize the output power. ${ }^{9}$ The emission frequency of the second-order DFB laser is about $2.55 \mathrm{THz}$, and the emission beam features a double-lobed pattern with a total divergence angle (FWHM) of $12 \times 36^{\circ}$. Fig. 5(b) presents the $L-I-V$ curves of a second-order DFB laser and a THz-MOPA-QCL in which the MO and the PA sections are equally biased. Both devices are measured at $20 \mathrm{~K}$ in pulsed mode with a repeat frequency of $25 \mathrm{kHz}$ and a pulse width of $1 \mu \mathrm{s}$. The maximum output power of the THzMOPA-QCL is $1.38 \mathrm{~mW}$, about twice that of the DFB laser $(0.60 \mathrm{~mW})$. The slope efficiency and the wall-plug-efficiency of the THz-MOPA-QCL are, respectively, $4.2 \mathrm{~mW} / \mathrm{A}$ and $0.03 \%$, higher than those of the second-order DFB laser.

Since the laser performances are closely related to the type of the active region, it is interesting to compare the THzMOPA-QCLs with the ever reported high performance singlemode THz-QCLs based on a similar bound-to-continuum active region and a metal-metal waveguide. ${ }^{9,10,17}$ We note that the maximum output power of the THz-MOPA-QCLs is in between those of the second-order DFB lasers and THz-QCLs with a graded photonic heterostructure (GPH) resonator. ${ }^{9,10}$ The slope efficiency and the wall-plug-efficiency of the THzMOPA-QCLs are close to the second-order DFB lasers.

Improving the output power and the power efficiency of THz-MOPA-QCLs is expectable by modifying the MO and the PA structures. By using a semiconductor DFB grating, formed by shallowly etching the active region and covering it by the top metallization, one can flexibly tune the coupling coefficient of the first-order DFB laser and thus significantly improve the MO output. In addition, exploiting a passive diffraction grating will further decrease the residual reflection and enable a longer preamplifier.

In conclusion, we have realized monolithically integrated THz-MOPA-QCLs and demonstrated stable generation and power amplification of single-mode emission. The amplification factor is about 5 , and the output power is approximately twice that of the standard second-order DFB lasers fabricated from the same material. Our work points out a direction for the realization of single-mode THz-QCLs with high output power and good beam quality. 
This work is partially supported by the National Natural Science Foundation of China under Grant No. 61574149, the Key Project of Chinese National Programs for Research and Development under Grant Nos. 2016YFB0402303 and 2016YFA0202200, "The Hundred Talents Program" of CAS, and the "Shanghai Pujiang Program" under Grant No. 14PJ1409700. The Engineering and Physical Sciences Research Council (EPSRC), UK (COTS programme EP/ J017671/1), and the Royal Society and Wolfson Foundation are also acknowledged. R.C. acknowledges support from the ERC "GEM" grant (Grant Agreement No. 306661) and from the FET project ULTRA-QCL. P-B. V. acknowledges support from the the FET project ULTRA-QCL.

${ }^{1}$ R. Köhler, A. Tredicucci, F. Beltram, H. Beere, E. H. Linfield, A. G. Davies, D. A. Ritchie, and R. C. Iotti, F. Rossi, Nature 417, 156 (2002).

${ }^{2}$ B. S. Williams, Nat. Photonics 1, 517 (2007).

${ }^{3}$ M. S. Vitiello, G. Scalari, B. Williams, and P. D. Natale, Opt. Express 23, 5167 (2015).

${ }^{4}$ C. Sirtori, S. Barbieri, and R. Colombelli, Nat. Photonics 7, 691 (2013).

${ }^{5}$ M. I. Amanti, M. Fischer, G. Scalari, M. Beck, and J. Faist, Nat. Photonics 3, 586 (2009).

${ }^{6}$ M. I. Amanti, G. Scalari, F. Castellano, M. Beck, and J. Faist, Opt. Express 18, 6390 (2010).

${ }^{7}$ L. Mahler, A. Tredicucci, F. Beltram, C. Walther, J. Faist, H. E. Beere, and D. A. Ritchie, Appl. Phys. Lett. 96, 191109 (2010).

${ }^{8}$ Y. Chassagneux, R. Colombelli, W. Maineult, S. Barbieri, H. Beere, D. Ritchie, S. P. Khanna, E. H. Linfield, and A. G. Davies, Nature 457, 174-178 (2009).

${ }^{9}$ G. Xu, R. Colombelli, S. P. Khanna, A. Belarouci, X. Letartre, L. Li, E. H. Linfield, A. G. Davies, H. E. Beere, and D. A. Ritchie, Nat. Commun. 3, 952 (2012).
${ }^{10}$ G. Xu, Y. Halioua, S. Moumdji, R. Colombelli, H. E. Beere, and D. A. Ritchie, Appl. Phys. Lett. 102, 231105 (2013).

${ }^{11}$ G. Xu, L. Li, N. Isac, Y. Halioua, A. Giles Davies, E. H. Linfield, and R. Colombelli, Appl. Phys. Lett. 104, 091112 (2014).

${ }^{12}$ T.-Y. Kao, Q. Hu, and J. L. Reno, Appl. Phys. Lett. 96, 101106 (2010).

${ }^{13}$ T.-Y. Kao, J. L. Reno, and Q. Hu, Nat. Photonics 10, 541 (2016).

${ }^{14}$ Y. Halioua, G. Xu, S. Moumdji, L. Li, J. Zhu, E. H. Linfield, A. G. Davies, H. E. Beere, D. A. Ritchie, and R. Colombelli, Opt. Express 23, 6915 (2015).

${ }^{15}$ M. Brandstetter, C. Deutsch, M. Krall, H. Detz, D. C. MacFarland, T. Zederbauer, A. M. Andrews, W. Schrenk, G. Strasser, and K. Unterrainer, Appl. Phys. Lett. 103, 171113 (2013).

${ }^{16}$ L. Xu, C. A. Curwen, P. W. C. Hon, Q.-S. Chen, T. Itoh, and B. S. Williams, Appl. Phys. Lett. 107, 221105 (2015).

${ }^{17}$ J. A. Fan, M. A. Belkin, and F. Capasso, Opt. Express 14, 11672 (2006).

${ }^{18}$ H. Wenzel, S. Schwertfeger, A. Klehr, D. Jedrzejczyk, T. Hoffmann, and G. Erbert, Opt. Lett. 37, 1826 (2012).

${ }^{19}$ S. O'Brien, D. F. Welch, R. A. Parke, D. Mehuys, K. Dzurko, R. J. Lang, R. Waarts, and D. Scifres, IEEE J. Quantum Electron. 29, 2052 (1993).

${ }^{20}$ M. Troccoli, C. Gmachl, F. Capasso, D. L. Sivco, and A. Y. Cho, Appl. Phys. Lett. 80, 4103 (2002).

${ }^{21}$ P. Rauter, S. Menzel, A. K. Goyal, B. Gökden, C. A. Wang, A. Sanchez, G. W. Turner, and F. Capasso, Appl. Phys. Lett. 101, 261117 (2012).

${ }^{22}$ B. Hinkov, M. Beck, E. Gini, and J. Faist, Opt. Express 21, 19180 (2013).

${ }^{23}$ C. Mauro, R. P. Green, A. Tredicucci, F. Beltram, H. E. Beere, and D. A. Ritchie, J. Appl. Phys. 102, 063101 (2007).

${ }^{24}$ Y. Ren, R. Wallis, Y. D. Shah, D. S. Jessop, R. Degl'Innocenti, A. Klimont, V. Kamboj, H. E. Beere, and D. A. Ritchie, Appl. Phys. Lett. 105, 141102 (2014).

${ }^{25}$ S. Barbieri, J. Alton, H. E. Beere, J. Fowler, E. H. Linfield, and D. A. Ritchie, Appl. Phys. Lett. 85, 1674 (2004).

${ }^{26}$ E. D. Palik, in Handbook of Optical Constants of Solids, edited by E. D. Palik (Academic, San Diego, 1985), Part II, pp. 429-443. 